\title{
Historical Development and Perspectives of the Series Metal Ions in Life Sciences*
}

It is old wisdom that metals are indispensable for life. Indeed, several of them, like sodium, potassium, and calcium, are easily discovered in living matter. However, the role of metals and their impact on life remained largely hidden until inorganic chemistry and coordination chemistry experienced a pronounced revival in the 1950s. The experimental and theoretical tools created in this period and their application to biochemical problems led to the development of the field or discipline now known as Bioinorganic Chemistry, Inorganic Biochemistry, or more recently also often addressed as Biological Inorganic Chemistry.

By 1970 Bioinorganic Chemistry was established and further promoted by the book series Metal Ions in Biological Systems founded in 1973 (edited by H. S., who was soon joined by A. S.) and published by Marcel Dekker, Inc., New York, for more than 30 years. After this company ceased to be a family endeavor and its acquisition by another company, we decided, after having edited 44 volumes of the MIBS series (the last two together with R. K. O. S.) to launch a new and broader minded series to cover today's needs in the Life Sciences. Therefore, the Sigels' new series is entitled

\section{Metal Ions in Life Sciences.}

After publication of 16 volumes (since 2006) with various publishers during the past 10 years, we are happy to join forces now in this still growing endeavor with Walter de Gruyter GmbH, Berlin, Germany, a most experienced Publisher in the Sciences.

The development of Biological Inorganic Chemistry during the past 40 years was and still is driven by several factors; among these are (i) attempts to reveal the interplay between metal ions and hormones or vitamins, etc., (ii) efforts regarding the understanding of accumulation, transport, metabolism, and toxicity of metal ions, (iii) the development and application of metal-based drugs,

* Reproduced with some alterations by permission of John Wiley \& Sons, Ltd., Chichester, UK (copyright 2006) from pages v and vi of Volume 1 of the series Metal Ions in Life Sciences (MILS-1). 
(iv) biomimetic syntheses with the aim to understand biological processes as well as to create efficient catalysts, (v) the determination of high-resolution structures of proteins, nucleic acids, and other biomolecules, (vi) the utilization of powerful spectroscopic tools allowing studies of structures and dynamics, and (vii), more recently, the widespread use of macromolecular engineering to create new biologically relevant structures at will. All this and more is reflected in the volumes of the series Metal Ions in Life Sciences.

The importance of metal ions to the vital functions of living organisms, hence, to their health and well-being, is nowadays well accepted. However, in spite of all the progress made, we are still only at the brink of understanding these processes. Therefore, the series Metal Ions in Life Sciences links coordination chemistry and biochemistry in their widest sense. Despite the evident expectation that a great deal of future outstanding discoveries will be made in the interdisciplinary areas of science, there are still "language" barriers between the historically separate spheres of chemistry, biology, medicine, and physics. Thus, it is one of the aims of this series to catalyze mutual "understanding".

It is our hope that Metal Ions in Life Sciences continues to prove a stimulus for new activities in the fascinating "field" of Biological Inorganic Chemistry. If so, it will well serve its purpose and be a rewarding result for the efforts spent by the authors.

\author{
Astrid Sigel and Helmut Sigel \\ Department of Chemistry, Inorganic Chemistry \\ University of Basel, CH-4056 Basel, Switzerland \\ Roland K. O. Sigel \\ Department of Chemistry \\ University of Zürich, CH-8057 Zürich, Switzerland
}

October 2005 and September 2016 